\title{
Search for subthreshold neutron production in Be
}

\author{
R. E. Chrien, D. E. Alburger, R. J. Sutter, and J. F. Wishart \\ Brookhaven National Laboratory, Upton, NY 11973
}

(Dated: September 15, 2004)

\begin{abstract}
We have searched for neutrons from the 3-body photon-induced reaction ${ }^{9} \mathrm{Be}+\gamma \rightarrow \alpha+\alpha+\mathrm{n}$ using bremsstrahlung produced by electrons from a 2-MV Van de Graaff. The target was located within a block of beryllium surrounded by an array of ${ }^{3} \mathrm{He}$ proportional counters embedded in paraffin. Based on energy and intensity calibrations of the accelerator and detector using the ${ }^{9} \mathrm{Be}+\gamma \rightarrow{ }^{8} \mathrm{Be}+n$ reaction, an upper limit of $93 \mathrm{nb}(4 \sigma)$ was placed on the cross section for neutron production between the 3-body and 2-body thresholds. This value is substantially below a previously experimental result using photoexcitation by a ${ }^{142} \mathrm{Pr}$ gamma source and also below an earlier theoretical estimate. We suggest that bremsstrahlung due to beta rays between $1665 \mathrm{keV}$ and the $2160-\mathrm{keV}$ end-point of the ${ }^{142} \mathrm{Pr}$ beta-ray spectrum could account for the photoneutron yield in the 3-body region that had previously been attributed to ${ }^{142} \operatorname{Pr}$ gamma rays.
\end{abstract}

PACS numbers:

\section{INTRODUCTION}

Beryllium is an interesting nucleus from three different points of view. It has the lowest neutron separation energy of any stable nuclide and can be thought of as a pair of $\alpha$-particles bound by an easily detachable neutron; it may be, therefore, a good test bed for a nuclear 3-body force. Its role in stellar nucleosynthesis has been recognized because of $\mathrm{N}=8$ gap in the chart of the nuclides. Finally, because of the availability of electron storage rings coupled to a laser source, Be can provide a unique, nearly monoenergetic source of neutrons near 1.6 MeV. For all these reasons, we have recently attempted to reexamine the threshold photodisintegration of ${ }^{9} \mathrm{Be}$.

The well-known 2-body break-up of Be via the photonuclear reaction ${ }^{9} \mathrm{Be}+\gamma \rightarrow{ }^{8} \mathrm{Be}+n$ has a threshold energy of $1665.4 \mathrm{keV}[1]$. The resulting ${ }^{8} \mathrm{Be}$ immediately breaks up into two $\alpha$ particles with a lifetime of $0.07 \mathrm{fs}$ and a combined kinetic energy of $91.9 \mathrm{keV}$. The presence of a resonance just above threshold results in a sharp increase in the cross section to a value of about $1 \mathrm{mb}$ [2] and makes this reaction useful for the calibration of lowenergy electron accelerators. Furthermore, the 3-body break-up of $\mathrm{Be}$ via the ${ }^{9} \mathrm{Be}+\gamma \rightarrow \alpha+\alpha+\mathrm{n}$ reaction is of theoretical interest as a measure of 3-body nuclear forces.

In a theoretical treatment of the photonuclear process Salyers[3], estimated a cross section of $0.2 \mu \mathrm{b}$ for the 3 body break-up near 2-body threshold. Some years later (1983) Fujishiro et al. [5] claimed to have actually observed neutrons from the ${ }^{9} \mathrm{Be}+\gamma \rightarrow \alpha+\alpha+\mathrm{n}$ reaction induced by $1575.7-\mathrm{keV} \gamma$ rays from ${ }^{142} \mathrm{Pr}$ decay. The neutron yield decayed with the 19-hr half-life of ${ }^{142} \mathrm{Pr}$ and a cross section of $0.40(18) \mu \mathrm{b}$ was derived. This was deemed in fair agreement with the theoretical estimate by Salyers of $0.2 \mu \mathrm{b}$.

However, there are good reasons for doubting the results of Ref. 5. Although they noted that the average energy of the ${ }^{142} \mathrm{Pr}$ ground-state $\beta$ rays is $833 \mathrm{keV}$, well below the 2-body threshold, they concluded that the bremsstrahlung from $\beta$-rays should not play a role in the neutron yield. However, the $\beta$-ray end-point energy to the ${ }^{142} \mathrm{Nd}$ ground state is at $2160 \mathrm{keV}[1]$ which is 495 $\mathrm{keV}$ above the 2-body threshold. Further, the $\beta$-ray decay branching ratio is $96.3 \%$ whereas the $\gamma$-ray branch to the $1567 \mathrm{keV}$ state is only $3.7 \%$; therefore the electron intensity is 26 times stronger than the $\gamma$ intensity. It is entirely plausible that the $\beta$ rays could indeed produce bremsstrahlung above the 2-body threshold and could account for the observed neutron yield.

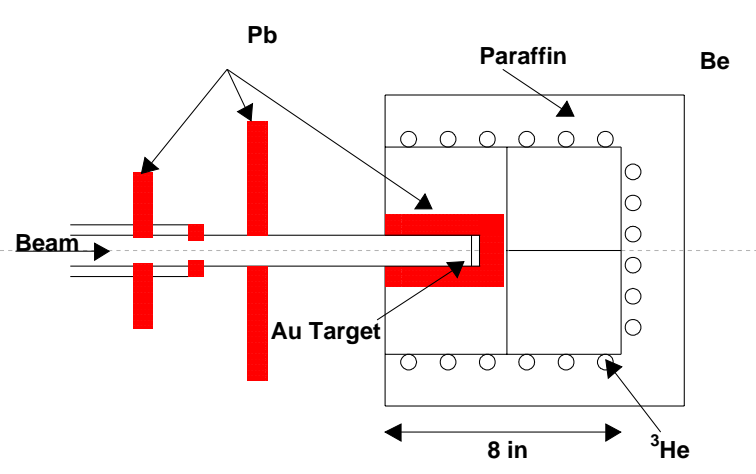

FIG. 1: A schematic of the experimental apparatus. The detector array is shown positioned just downstream of the accelerator tube end.

To investigate neutron production in beryllium, we have chosen to use bremsstrahlung from a gold target bombarded with electrons of variable energy. Although the bremsstrahlung spectrum is a continuum, this method has the advantage of a controlled, welldefined upper limit to the photon energy distribution and no interference by the 2 -body reaction can occur in the 3 -body region. We searched for neutrons from the ${ }^{9} \mathrm{Be}+\gamma \rightarrow \alpha+\alpha+\mathrm{n}$ reaction at photon energies below, above, and between the 3-body and 2-body thresholds. 


\section{EXPERIMENTAL PROCEDURES AND RESULTS}

Figure 1 is a schematic drawing of the experimental arrangement for the neutron measurements. A continuous electron beam originates from the 2-MV Van de Graaff accelerator in the BNL Chemistry Dept. After collimation the beam strikes a $0.13-\mathrm{mm}$ thick gold target soldered to a $4.7-\mathrm{mm}$ thick brass plug. The target tube is enclosed in a $2.5-\mathrm{cm}$ thick $\mathrm{Pb}$ sleeve in order to discriminate strongly against low-energy bremsstrahlung. The gold target is at the center of an assembly of 200 machined Be blocks, each $2 \times 2 \times 1 / 2$ inches in size, which were available from previous reactor experiments. These were aligned and epoxyed together to form large blocks with dimensions of $4 \times 4$ inches square and 3.5 or 4 inches thick, stacked closely around the target, for a total weight of about $13.5 \mathrm{kgm}$. This assembly was in turn surrounded, on five sides, by a ${ }^{3} \mathrm{He}-$ proportional counter array embedded in paraffin.

For neutron detection, a number of ${ }^{3} \mathrm{He}-$ filled proportional counters used in previous slow neutron timeof-flight experiments at Brookhaven were saved from a planned discard. Thirty good tubes were used in the final set-up, where they were seated in slots machined in $4-\mathrm{cm}$ thick paraffin slabs. The tubes were $1 / 2$ " in diameter, 13 " long, and filled with ${ }^{3} \mathrm{He}$ gas at 8 atm pressure. The tubes were in groups of 6 on 5 sides of the Be assembly, 3 of which are shown in Fig.1. The efficiency of the detector was measured by placing a $3 \mathrm{mCi}{ }^{241} \mathrm{Am}$-Be neutron source, assayed as emitting 6000 neutrons/sec, at the center of the array. With the discrimination level just above electronic noise, an efficiency of $\sim 25 \%$ was achieved. In order to reduce the incidence of bremsstrahlung on the counters from sources other than the gold target, the beam collimator was made of $\mathrm{Al}$, and $\mathrm{Pb}$ shields were placed as shown in Fig. 1.

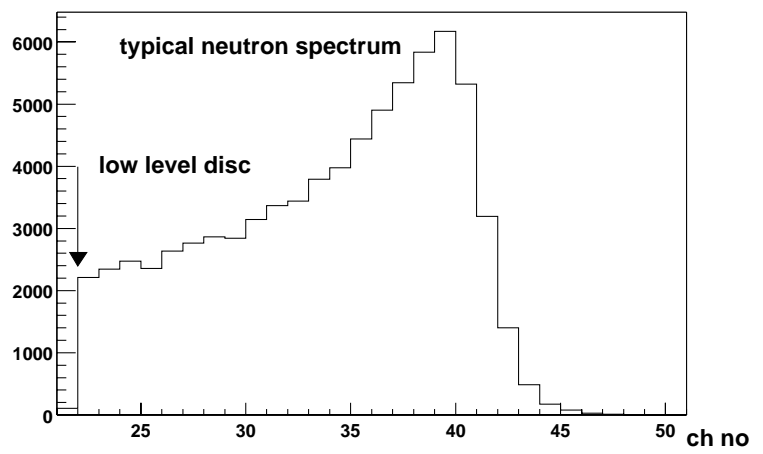

FIG. 2: A typical counter response at the applied high voltage of 1350 volts used in the experiment. Each of the $30{ }^{3} \mathrm{He}$ proportional counters had a very similar response.

Initial tests with an electron beam on the target and with all 30 tubes connected to a single slow preamplifier, however, showed severe count-rate limitations due to the pile-up of small pulses resulting from the detector response to bremsstrahlung, indicating the need for a faster electronic system for the array. Futhermore, the ${ }^{3} \mathrm{He}$ counters presented special problems because of their unfavorable gas collection geometry and large wall effect, as is well known[7,8] in neutron spectroscopy with such detectors. A compromise between the long time $(\approx 1$ ms) for optimum charge collection and uniform pulse response, and the rate handling capability necessary for the array, led to a limitation of $\approx 5 \mu$ sec on pulse length. The counters were paired to reduce the number of electronic channels required and coupled into the inputs of a 16-channel transconductance preamplifier originally designed for the neutral meson spectrometer constructed at Los Alamos[9]. Outputs from the preamplifier were transformer-coupled into a NIM-based, wide-band dccoupled amplifying system. The resulting signal had a characteristic rise-time of $\approx 0.2 \mu$ sec and a length of $\approx 5$ $\mu$ sec. Counting rates of tens of $\mathrm{kHz}$ could thus be tolerated with negligible dead time.

At the adopted bias on the counters of $1350 \mathrm{~V}$, the typical amplitude spectrum due to neutrons from bremsstrahlung photoneutrons is shown in Fig. 2. The discrimination level, shown by the arrow, was selected to suppress the machine-dependent background, almost entirely due to bremsstrahlung incident on the detectors. We estimate the neutron efficiency-acceptance product during our runs at $\sim 12 \%$.

An electron beam current of $\approx 100 \mathrm{nA}$ from the Van de Graaff was selected for the final runs as giving a strong yield of neutrons above the 2-body threshold and a low machine-dependent background due to the bremsstrahlung sensitivity of the detectors. At the current chosen about two-thirds of the background was machine-independent. To establish the energy calibration of the Van de Graaff, a set of yield curves were taken throughout the experiment; a linear extrapolation to zero of the square root of the count rate was taken to relate the accelerator voltage to the threshold energy of 1.6654 $\mathrm{MeV}$.

The experimental yield was compared to a calculated product of a thick target bremsstrahlung spectrum[10] and the photoneutron cross section of beryllium, taken from the measurements of Ref. [2]. The agreement between simulated and experimental yield established a reliable cross section scale.

\section{DISCUSSION}

From the photoneutron measurements shown in Fig. 3 and the cross section calibration previously described, the observed rate after backbround subtraction, in the region between 2- and 3-body thresholds, is measured to be $0.015 \pm 0.0072 / \mathrm{sec}$. This corresponds to a cross section of $32 \pm 15 \mathrm{nb}$. Thus we may place, at a 4 sigma confidence level, an upper limit of $93 \mathrm{nb}$ on the production cross section. Our measurement suggests that the 


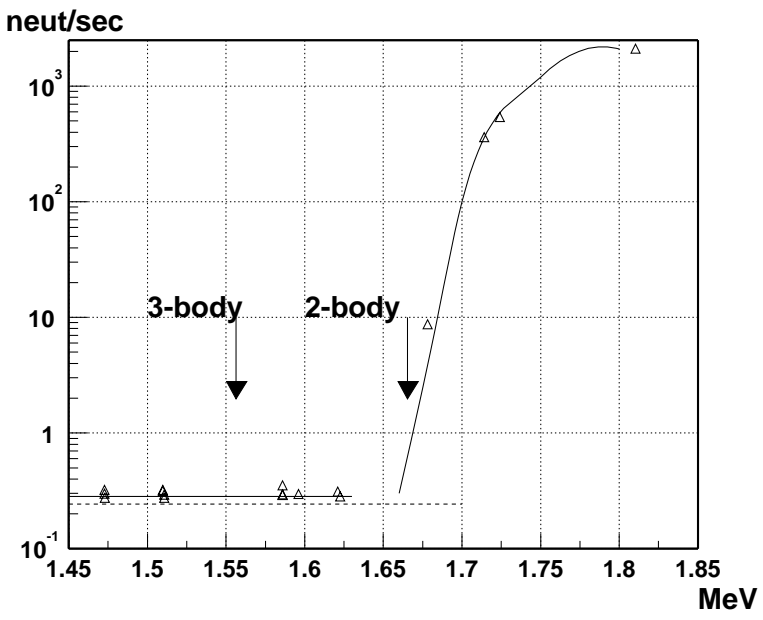

FIG. 3: The photoneutron rate from all 30 detectors, recorded as a function of bremsstrahlung endpoint over the electron energy range from 1.45 to $1.85 \mathrm{MeV}$. The region between the 2 - and 3-body thresholds is indicated. The solid line shows the total machine-on background, while the dashed line indicates machine-off background, believed to be almost totally due to cosmic rays. The curve drawn above the 2 -body threshold is based on our calculation as described in the text.

3-body admixture in the nuclear 2-body force is quite small.

This value may be compared to a prediction $0.2 \mu \mathrm{b}$ of Salyers and the reported $0.4 \mu \mathrm{b}$ of Fujishiro et al.[5]. They used a radioactive source for the photons initating the reaction.

To test our hypothesis that the results of ref. [5] were affected by $\beta$-ray induced bremsstrahlung, we measured photon yields from the Van de Graaff as a function of angle. This was done by replacing the neutron detector array by a goniometer table on which was mounted a 3 by 3 -inch $\mathrm{NaI}$ detector. A series of angle and energy measurements of the accelerator output was taken.

These data were corrected for absorbtion and detector efficiency to determine the total yield of photons above $1665 \mathrm{keV}$ at each beam energy out to $80^{\circ}$. The NaI detector efficiency,the attenuation in the brass holder and lead beam filter were all accounted for to produce the corrected yield shown in Fig.4.

Also shown in this figure is the upper end of the ${ }^{142} \mathrm{Pr}$ ground-state $\beta$ spectrum, calculated for an allowed transition with an end-point energy at $2160 \mathrm{keV}$. The photon yield multiplied by the $\beta$-fraction leads to an expected yield of $3.4 \times 10^{-5}$ photons per ${ }^{142} \mathrm{Pr}$ disintegration. It has been learned[11] from one of the authors of Ref. [5] that the ${ }^{142} \mathrm{Pr}$ source was made by irradiating pure $\mathrm{Pr}$ metal in a reactor. Since $\operatorname{Pr}$ has $\mathrm{Z}=59$ and gold has $\mathrm{Z}=79$, a $\mathrm{Z}^{2}$ correction leads to a prediction that ${ }^{142} \operatorname{Pr} \beta$ rays on $\operatorname{Pr}$ would produce $1.9 \times 10^{-5}$ photons per decay above the 2-body threshold. In the experiments of Ref. 5 the reported 3-body cross section was smaller than the 2 -body cross section by a factor of 2200 . In order to re-

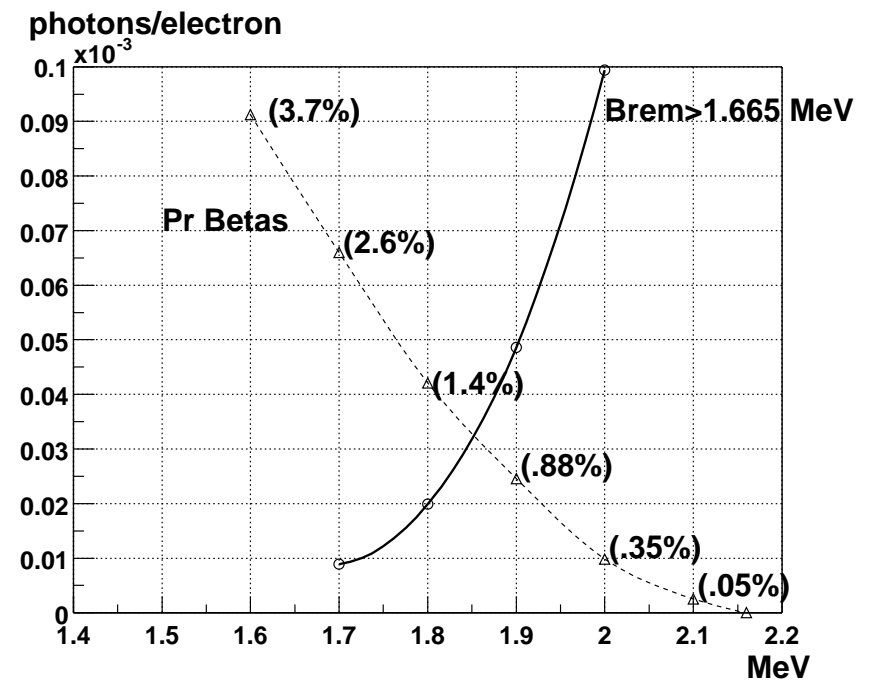

FIG. 4: The solid curve shows the photons per incident electron above $1665 \mathrm{keV}$ at the four beam energies. The dashed curve is the constructed plot of an allowed ${ }^{142} \mathrm{Pr}$ beta-ray spectrum from $1.5 \mathrm{MeV}$ to an end-point at $2160 \mathrm{keV}$. The fraction of betas exceeding a given energy is indicated on the curve.

sult in that yield, since the branch to the $1576-\mathrm{keV}^{142} \mathrm{Nd}$ state is $3.7 \%$, the photon intensity for gamma rays above the 2-body threshold would be $0.037 / 2200=1.6 \times 10^{-5}$ per decay. This is consistent with our prediction. The uncertainty in the result of Ref. 5 is $45 \%$ and our own predicted value is probably good to no better than $50 \%$. Thus, the close agreement is surely fortuitous.

Briefly, our conclusion is that the photon intensity for gamma rays above the 2-body threshold would be $1.6 \times$ $10^{-5}$ per decay. That value is quite adequate to account for the neutron production of Ref. [5].

This cross section limit represents an average over the interval between the 2-body and 3-body thresholds. There is no theoretical calculation of the energy dependence of the process; however one might expect that the alpha particle barrier penetrability is strongly energy dependent and that the cross section would be largest at the 2-body threshold.

A completely different approach to this problem would be to irradiate beryllium with monoenergetic photons of variable energy using the laser-induced Compton backscattered gamma rays technique, as described in Ref. [12]. This might be feasible if a source with sufficient gamma-ray intensity to scan the 3-body reaction region could be developed. We note that there are current attempts to implement this method to produce a neutron source which is very nearly monoenergetic. Such a device has been created at at the electron storage ring at the Electrotechnical Laboratory at Ibaraki, and is planned for the synchrotron radiation source spring8 at Osaka. Such devices will bring neutrons to complement material science research at such facilities.

If a significant 3-body reaction in Be photodisintegra- 
tion were to exist, it might merit consideration for its role in stellar nucleosynthesis, a topic of increasing interest in fundamental nuclear physics. The $\mathrm{N}=8$ gap in stellar nucleosynthsis is bridged by a triple $\alpha$ reaction leading to ${ }^{12} C$. However, in a neutron-rich environment, such as in a core-collapsed supernova, a 3-body $\alpha(\alpha n, \gamma)$ may compete with the triple $\alpha$ process[12]. For this reason, the search for a 3-body effect in Be photodisintegration remains a challenging opportunity in low-energy nuclear research.

\section{ACKNOWLEDGEMENTS}

The authors wish to express their appreciation to Maurice Goldhaber, who suggested this research problem and provided continual encouragement. We are grateful for the assistance of Stephen Howell and Harold Schwarz of the Brookhaven Chemistry Dept. The ColliderAccelerator Department's High Energy Equipment Pool (HEEP) provided much of the electronic modules used in the experiment. We also thank Kathryn Kolsky for the loan of a computer for data acquisition and analysis, and for help in its use. This work was carried out at Brookhaven National Laboratory under contract DEAC02-98CH10886 with the U.S. Department of Energy and supported by its Division of Chemical Sciences, Office of Basic Energy Sciences.
[1] National Nuclear Data Center, Brookhaven National Laboratory, 2004. All transition energies and thresholds were taken from latest compilations at the NNDC.

[2] B. L. Berman, R. L. Van Hemert, and C. D. Bowman, Phys. Rev. 163, 9581967

[3] A. Salyers, Phys. Rev. C2, 16531970

[4] D. E. Alburger, Phys. Rev. 8812571952

[5] M. Fujishiro, K. Okamoto, and T. Tsuijimoto, Can. J. Phys. 6016721983

[6] H. Utsunomiya et al. Phys. Rev. C63 018801-1 2000;

[7] S. Shalev, Z. Fishelson, and J. M. Cuttler, Nucl. Inst. Meth 712921969
[8] K. Zeitelback et al. Internal Report 09-02-01 Tech. U. Munchen, 2001 (This report illustrates nicely the problems of signal processing from counters with large wall effects.)

[9] H. Baer et al. Nucl. Instr. Meth 1804451981

[10] M. Krmar et al Phys. Med. Biol. 38 533-544 1993; see also L. I. Schiff, Phys. Rev. 832521951

[11] Ken-ichi Okamoto, private communication, 2004

[12] H. Toyakawa et al. Ibaraki Electrotechnical Laboratory Report, 2000 (unpublished); see also H. Toyokawa et al., Nucl. Instr. and Meth. A422 95 1999; H. Ohgaki et al., Nucl. Instr. and Meth. A55 542000 